\title{
Numerical Simulation and Calibration of a Single Seam WAAM Process with a Commercial and an Open Source Software
}

\author{
Alexander Bauer ${ }^{1 *}$, Ronny Scharf², André Hälsig², Birgit Awiszus ${ }^{1}$ \\ ${ }^{1}$ Professorship of Virtual Production Engineering, Chemnitz University of Technology, Chemnitz, Germany \\ 2Professorship of Welding Engineering, Chemnitz University of Technology, Chemnitz, Germany \\ *corresponding author: a.bauer@mb.tu-chemnitz.de
}

\section{ABSTRACT}

This paper deals with the comparison of the FEM result quality between a commercial tool and an open source software with regard to the numerical simulation of a single seam WAAM process. For this purpose, an experimental WAAM process was conducted by a welding robot on a 1.4404 (316L) base plate with a 1.4404 (316L) welding wire, connected with the associated measurement equipment. Furthermore, the equivalent setup was transferred in the FEM tools Simufact Welding and Elmer FEM, using the same parameters for both simulations. The calculations showed that both programs were able to generate high quality results with good accordance to the temperature development within the experimental processes. In summary it can be said that both programs offer advantages and the decision on which tool has to be used depends on the requirements and the possibilities of the user.

Keywords: Simufact Welding, Elmer FEM, WAAM, additive manufacturing, welding simulation

\subsection{INTRODUCTION AND CURRENT STATE OF TECHNOLOGY}

The technology of additive manufacturing (AM) has become more important in current metal manufacturing environment. While high machine costs and long manufacturing time are obstacles according to the ramp up of AM technologies, recent innovations and technological advancements have led to a continuing improvement of efficiency and profitability, especially for prototypes and small quantities. In addition to the well-known powderbased AM process Selective Laser Melting (SLM), direct metal deposition processes such as Wire Arc Additive Manufacturing (WAAM) and 3D Plasma Metal Deposition (3DPMD) have become more important [1]. As a result of these rising demands and quantities, the need of promising forecasts is increasing similarly. For that reason, numerical simulation for AM processes is a very present topic in the current research project [2]. More commercial software developers have recognized the current market conditions and therefore, began to offer various programs specifically designed for the numerical simulation of AM processes. Famous examples are Simufact Additive (Simufact Engineering GmbH), ANSYS Additive Suite (ANSYS Inc.), AUTODESK NETFABB (Autodesk Inc.) or ABAQUS (Dassault Systèmes). Furthermore, open source programs like Elmer FEM and Code Aster have begun to offer functions for the simulation of AM processes. A key aspect for a successful implementation of FEM programs for AM applications is the reliability of the simulation results, including the geometrical and thermal accuracy of the model. A lot of research has been conducted in dealing with numerical simulation of various AM processes [3]. Since this paper is dealing with the WAAM process, the following short overview will be restricted to research topics around this technology.

In general, WAAM is an AM technology which produces components layer by layer, using the Gas-MetalArc-Welding (GMAW) technology. The biggest advantage of this technique is that large components can be manufactured in a short time period because of the higher deposition rate compared to other AM technologies [2]. However, WAAM components are also highly affected by unwanted distortions and residual stresses which are a major problem regarding to reproducible results and a reliable production. Therefore, the numerical simulation of WAAM components has become a significant factor for the prediction of distortions, unwanted side effects or process improvements, which also makes it a popular topic for current research studies [2], [4 -5]. In the following section, an overview about current research activities regarding to the numerical simulation of WAAM processes should be given.

Ding developed a new transient thermo-mechanical FE-model in ABAQUS to safe computational time while leaving the result quality on a good level of accordance. A reduction of up to $99 \%$ computational time was reached with still sufficient accordance regarding to the experiments [6]. Within [2], an improvement of the heat source modelling was tested in LS-DYNA. The aim was that the effect of power distribution between base plate and filler material can be considered for an improved numerical modelling. It was proven that the developed model showed a better accordance with regard to the experimental results than with the use of the standard Goldak's model. Another modification of the Goldak heat source model was implemented in ABAQUS by Ling. The hereby introduced improvement in which the feeding speed of the filler material wire was also considered for the 
numerical simulation, which led to an improved correctness of simulating the energy input [5]. For a reliable numerical simulation, Graf showed the importance of using the correct thermal material data, which were directly taken from the materials used for the experiments or at least completely identical samples with the same chemical composition [4]. In [7], arc-shaped and rectangular weld bead modellings were compared in MSC Marc and evaluated with accordance to experimental tests. Even when the arc shape represented a more detailed experimental geometry, it was detected that the rectangular shaped weld bead showed the same quality of agreement with the experiments regarding thermal effects and the distortion of the workpiece. Therefore, it was concluded that the use of a rectangular shape is more efficient because of a reduced computational time and an increased numerical stability. An optimization according to the computational time was examined by Huang. Within this research, a new finite element code was especially developed for AM simulations. The feasibility was later proven by the successful calculation of WAAM process, which showed a good agreement compared to the experiments [8]. Another publication which dealt with the increase of efficiency during the thermomechanical computation of a WAAM process by using aluminium alloys was [9]. The program used for the finite element simulation was ABAQUS, including a moving heat source model combined with the segment temperature function. On one hand, the used setup led to a reduced computational time; on the other hand, it led to a good agreement with error percentages below $20 \%$ according to distortion and residual stresses.

All in all, it can be recognized that most of the research paper dealt either with the improvement of the calculational accuracy with regard to distortion and temperature or with the increase of efficiency and the reduction of computational time. Next to the development of a reliable simulation model with good accordance compared to the experiments, the costs of the FEM tools should be considered. As specific AM programs are very expensive and mostly for professional use only, open source programs partly offer good alternatives for some numerical setups. Therefore, the calibration of a single seam WAAM experimental setup with two FEM simulation programs was investigated here. On one hand, the commercial FE tool Simufact Welding was used; on the other hand, the same process was modelled with the open source software Elmer FEM. Elmer is an open source multiphysics finite element solver that can be used to solve a wide variety of differential equations. Elmer has preinstalled solvers for numerous physical models from the fields of fluid dynamics, structural mechanics, electromagnetics, heat transfer and acoustics. In addition, the program can be extended using so-called User Defined Functions (UDF) and the Fortran programming language. With UDF's different heat sources (e.g., Goldak), complex material behaviour and additive material depositions can be implemented effectively. So, within this article, it should be clarified that Elmer FEM is able to provide good numerical results compared with commercial software solutions, if some aspects in the numerical modelling are considered.

\subsection{EXPERIMENTAL AND NUMERICAL SETUP}

\subsection{Experimental setup}

The experimental tests were conducted with the help of a robot-assisted welding system at Chemnitz University of Technology. This system consists of a 6-axis robot (KUKA KR 6) and the welding system (Fronius TransPuls Synergic, TPS 5000).
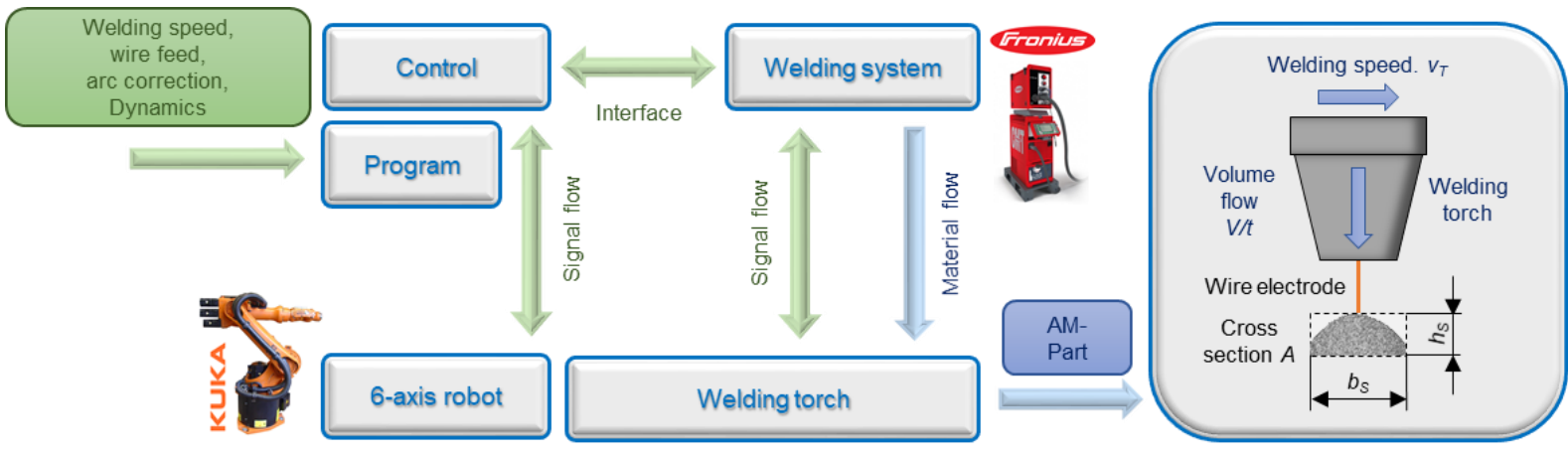

Figure 1. Signal and material flow of the robot-based WAAM welding system; welding parameters (welding speed $v_{T}$, volume flow per time $\left.V / t\right)$, which determine the layer width $b_{S}$ and height $h_{S}$

Figure 1 shows the block diagram of the complete welding system. The robot controller, which is the central processing unit of the robot system, interprets the robot programs and moves the manipulator along the programmed paths. A robot interface serves as a link between the robot controller and the welding system. The commands for the welding system and the movement of the robot are located in the corresponding program line of the robot program, which is interpreted by the controller and sent to the robot (movement) and the welding system (welding commands). The modified short arc welding process CMT used for this investigation is adjusted not only by the welding speed (traversing speed of the robot), but also by the parameters wire feed speed, arc correction and dynamics. The path coordinates for additive manufacturing are derived from a CAD model using 
a slicer and translated into executable welding programmes with a specially developed compiler and the programming language Python.

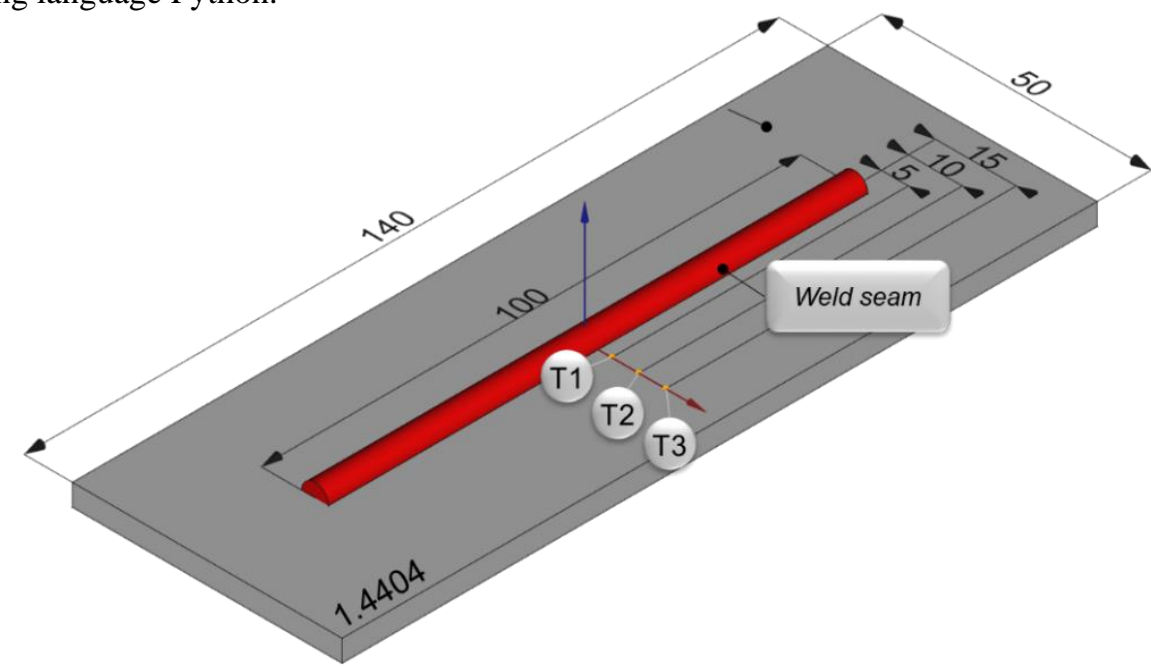

Figure 2. Straight weld seam of $100 \mathrm{~mm}$ length on substrate plate made of X2CrNiMo17-12-2 (316L), thermocouples type- $\mathrm{K}$ (T1, T2 and T3) for tactile temperature measurement during welding

Using the WAAM system described above, a straight seam with a length of $100 \mathrm{~mm}$ was welded on a substrate plate made of austenitic steel X2CrNiMo17-12-2, 316L (see Figure 2). The same material was selected as the filler material. The temperatures were measured in situ with three type-K thermocouples and a sampling rate of 5 $\mathrm{Hz}$ at the positions shown (T1, T2 and T3). The process was performed with a voltage of $14.7 \mathrm{~V}$, a current of 115 A and a welding speed of $0.0067 \mathrm{~m} / \mathrm{s}$.

\subsection{Numerical setup in Elmer FEM}

For the numerical simulation with Elmer FEM, a simple CAD model of a weld seam and a substrate plate was constructed and meshed with 50071 tetrahedron elements using the open source meshing tool Gmsh [10].

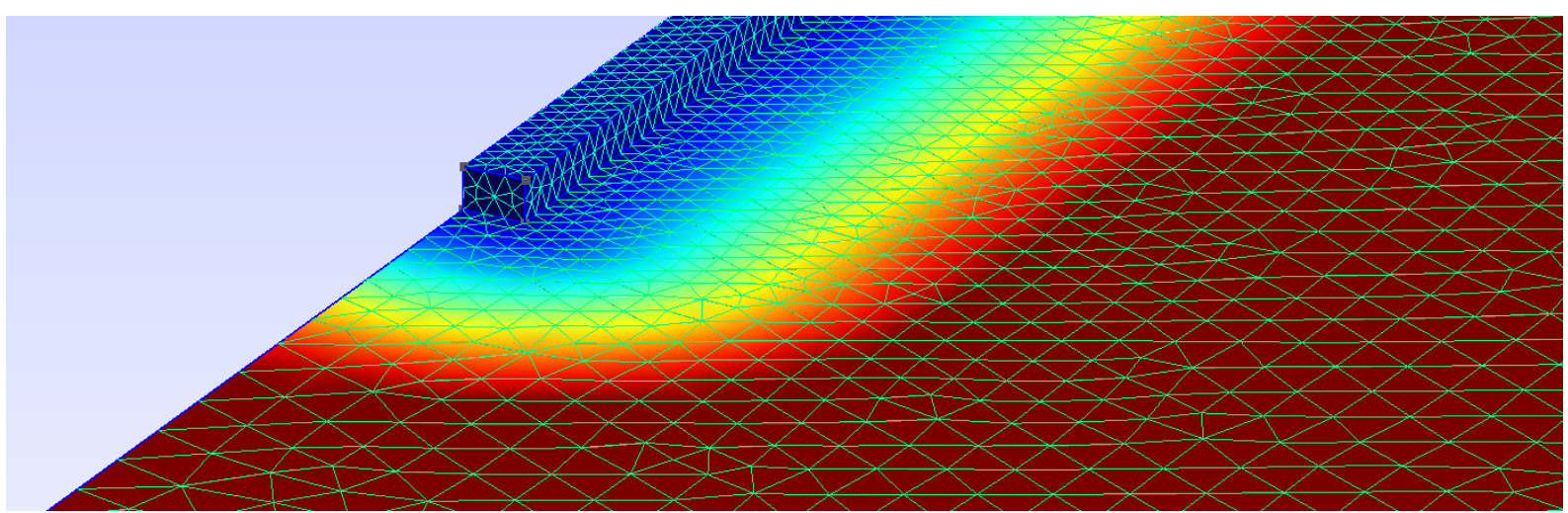

Figure 3. Mesh generation with Gmsh: Graded mesh consisting of 50071 tetrahedron elements with a continuous reduction of the edge length from $2 \mathrm{~mm}$ (red) to $0.5 \mathrm{~mm}$ (blue)

The element edge length was continuously reduced from $1 \mathrm{~mm}$ to $0.5 \mathrm{~mm}$ from the substrate plate to the weld seam to achieve a balance between accuracy and computation time (Figure 3). For efficiency reasons, the calculation was performed on the halved CAD model, taking advantage of the thermal symmetry. In addition, the mesh was partitioned into two parts using a Metis algorithm and solved in parallel on two CPU cores. Temperature-dependent material parameters can be passed to Elmer either by UDF or tabular. Fig. 4 shows the characteristic material values used for the simulation, which also map the transition between liquid and solid phase. 

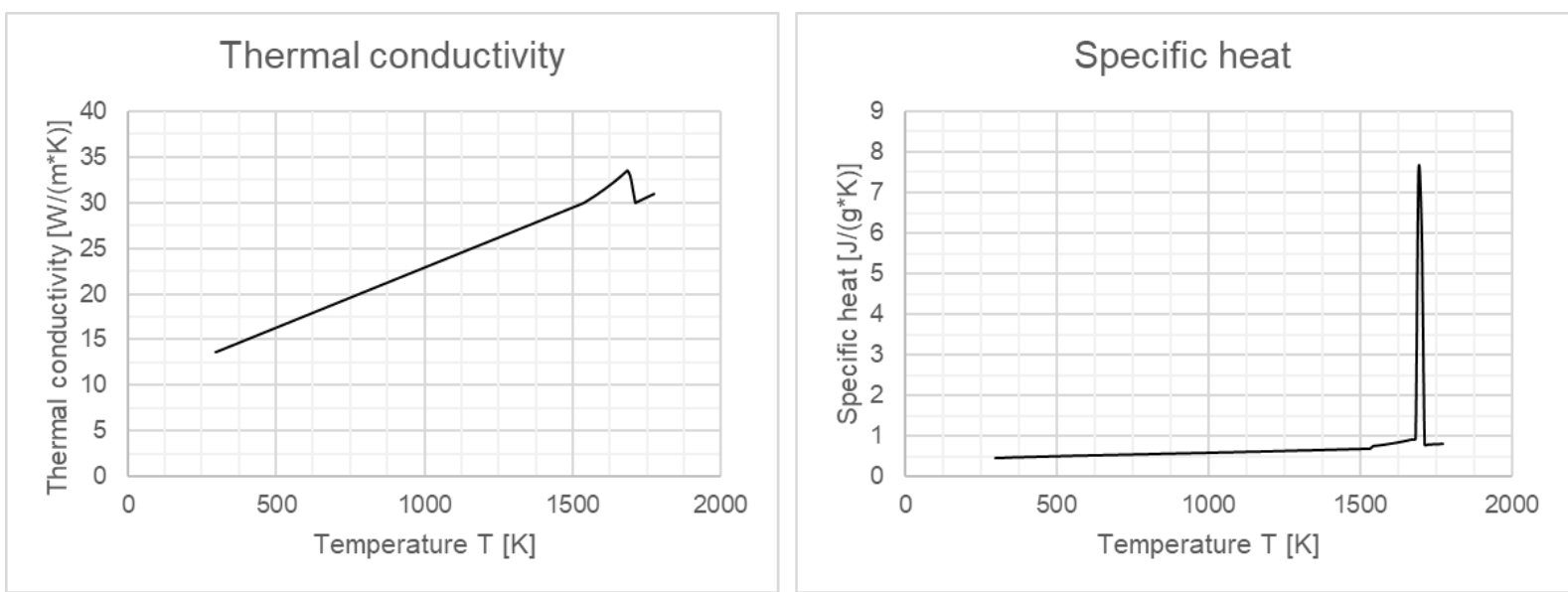

Figure 4. Temperature-dependent material behaviour, thermal conductivity and specific heat capacity for the test material X2CrNiMo17-12-2 (316L) from the materials database of JMatPro [11]

The implementation of Goldak heat source was done as a UDF with the parameters of front length $a_{f}=1.5 \mathrm{~mm}$, rear length $a_{r}=5.5 \mathrm{~mm}$, width $b=2.5 \mathrm{~mm}$ and depth $d=4.5 \mathrm{~mm}$. These values represent the Simufact default values for a weld height of $a=1 \mathrm{~mm}$ [12]. The heat transfer coefficients to the environment $\alpha_{T o p}$ and tool table $\alpha_{B o t t o m}$ were determined iteratively. The values $\alpha_{T o p}=15 \mathrm{~W} /\left(\mathrm{m}^{2} \cdot \mathrm{K}\right)$ and $\alpha_{B o t t o m}=150 \mathrm{~W} /\left(\mathrm{m}^{2} \cdot \mathrm{K}\right)$ produced a very good agreement with the experiment. The increased value for $\alpha_{\text {Bottom }}$ indicates moderate cooling of the tool table, which is in line with reality. The thermal transient calculation was performed with the standard values of Elmer's Heat Solver. The step size of the simulation corresponded to the sampling rate of the temperature measurement, so that the results could be easily compared. The additive material deposition was realized by continuously activating passive mesh elements (element birth strategy).

\subsection{Numerical setup in Simufact Welding 2020}

In Simufact Welding 2020, the WAAM setup was modelled with the direct energy deposition function and the solution was restricted to the thermal process, because the focus of the analyses laid on the thermal calibration. The thermal data were included as stated in section 2.2. All other parameters and dimensions were defined as given for the experimental setup in section 2.1. Therefore, a single weld seam was defined in the middle of the base plate for the direct energy deposition process. The general model setup in Simufact Welding can be seen in Figure 5.

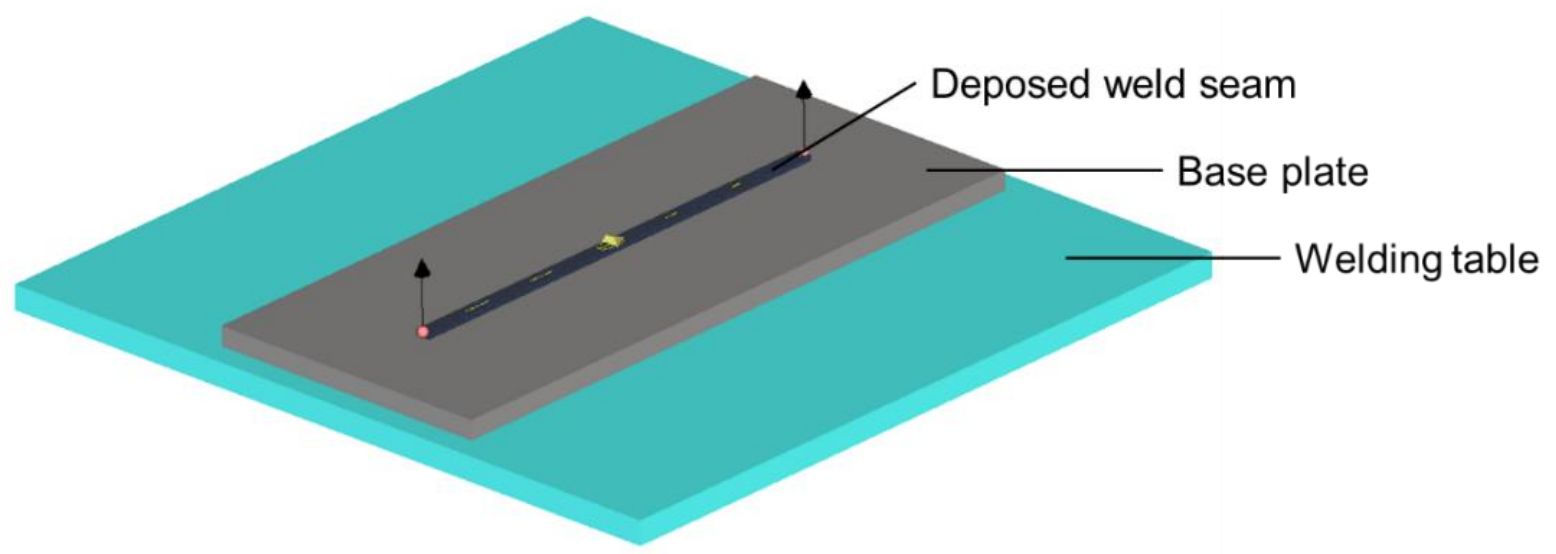

Figure 5. Model setup in Simufact Welding 2020

In Figure 5, it is also visible that no clamping was used to fix the base plate on the welding table because this is not necessary for a solely thermal calculation. The mesh of the base plate was provided by the sheet mesher function with a size of $1 \mathrm{~mm}$ and six elements in height direction, while the mesh of the weld seam was defined finer, with a size of $0.5 \mathrm{~mm}$ and three elements in height direction. The thermal conditions were applied separately for the base plate and the weld seam, which allowed them to define different parameters for the varying contact areas. For the base plate, the corresponding temperature data were implemented with a convective heat transfer coefficient of $15 \mathrm{~W} / \mathrm{m}^{2} \mathrm{~K}$ and a contact heat transfer coefficient of $225 \mathrm{~W} / \mathrm{m}^{2} \mathrm{~K}$. The values for the weld seam were 
given with a convective heat transfer coefficient of $15 \mathrm{~W} / \mathrm{m}^{2} \mathrm{~K}$ and a contact heat transfer coefficient of $10000 \mathrm{~W} / \mathrm{m}^{2} \mathrm{~K}$ (see Table 1 ).

Table 1: Mesh size and thermal boundary conditions for FEM simulations

\begin{tabular}{cccccc}
\hline \multicolumn{2}{c}{ Mesh Size } & \multicolumn{3}{c}{ Hear Transfer Coefficient } \\
\cline { 1 - 2 } \cline { 5 - 6 } base & weld & & to environment & base to table & base to weld \\
\cline { 1 - 2 } \cline { 5 - 6 } & $0.5 \mathrm{~mm}$ & & $15 \mathrm{~W} / \mathrm{m}^{2} \mathrm{~K}$ & $225 \mathrm{~W} / \mathrm{m}^{2} \mathrm{~K}$ & $10000 \mathrm{~W} / \mathrm{m}^{2} \mathrm{~K}$ \\
\hline
\end{tabular}

The contact heat transfer coefficient was chosen that high due to reason that for the melting of material, it can be seen one body emerges out of the two elements of weld seam and base plate. All in all, the heat transfer conditions were almost similar to the Elmer FEM simulation, except of the contact heat transfer of the base plate, which was selected slightly higher to achieve a better agreement. According to the power source, all parameters were used as within the experiments and the Goldak heat source model was defined as mentioned for Elmer FEM. For a better evaluation, tracking particles were defined within the numerical setup. The tracking particles were located at the same spots, where the thermocouples from the experiments were fixed (Figure 2). To fasten the computational time, a domain decomposition with two domains was used, while each of them was additionally subdivided into two corresponding calculating cores.

\subsection{RESULTS AND DISCUSSION}

Figure 6 shows the calculation result of Elmer FEM and Simufact Welding in comparison to the real experiment.
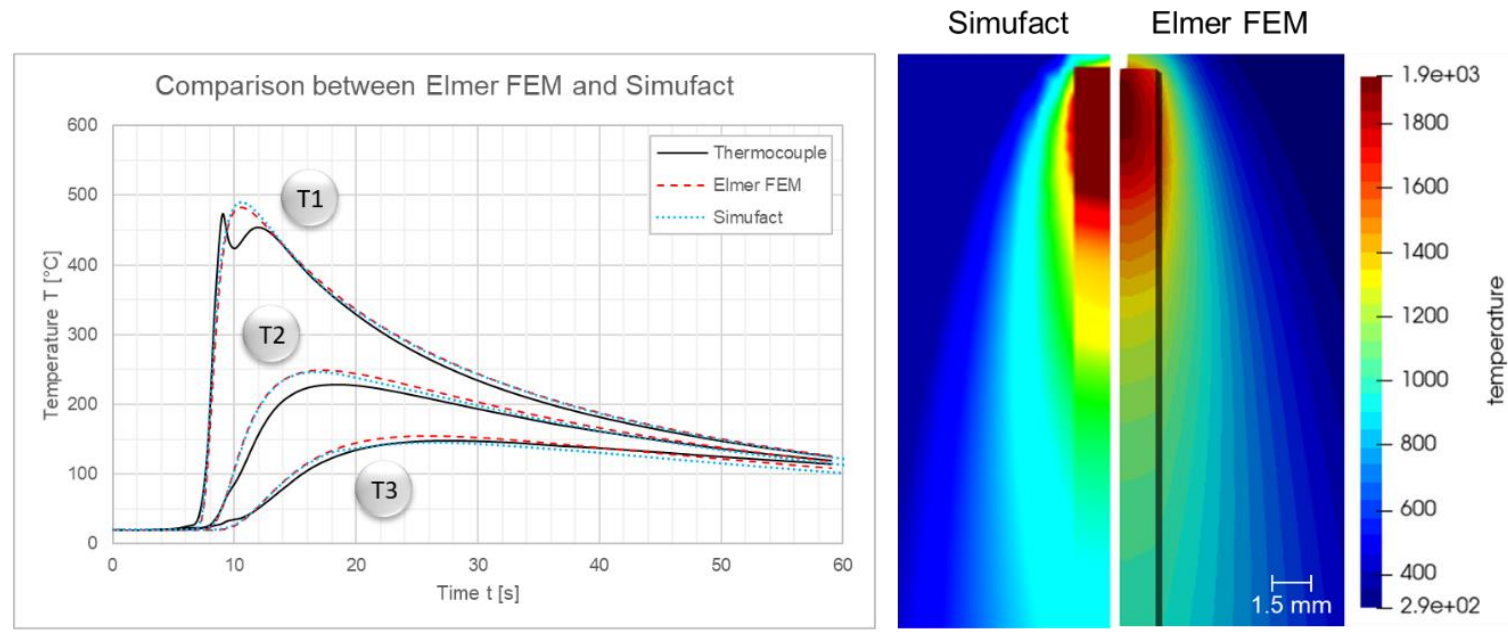

Figure 6. Comparison between experiment (black solid line), Elmer FEM (red string line) and Simufact

Welding (blue dotted line) at measuring points T1 to T3 (left), Visualization of the results, temperatures in Kelvin (right)

Both programs were able to solve the thermal problem in a comparable time (about 15 minutes). The calculation results of Elmer FEM and Simufact Welding both agree with the real measured temperatures to a large extent. In particular, the temperature curves of thermocouples T1 and T3 were very well reproduced. Only for thermocouple 2 (T2), the calculation produced an increased maximum which can be seen in both numerical simulation tools. The deviation from the experiment was approx. $20{ }^{\circ} \mathrm{C}$. The short temperature drop of thermocouple 1 (T1) after reaching the maximum temperature was generated by the used measuring system and consequently cannot be modelled with the thermal FEM.

After the calibration of the single seem was successfully completed, the numerical setup was expanded for the simulation of a complete hollow wall structure in Simufact Welding. Hereby, all the chosen parameters for the heat source and the thermal coefficients stayed exactly the same and only the trajectory was adjusted. The applied and calibrated setup showed promising results regarding to the simulation of a complete WAAM structure. Further investigations should show the accordance of the thermal and the geometrical development of the workpiece, which were influenced by thermal induced stresses (Figure 7). 

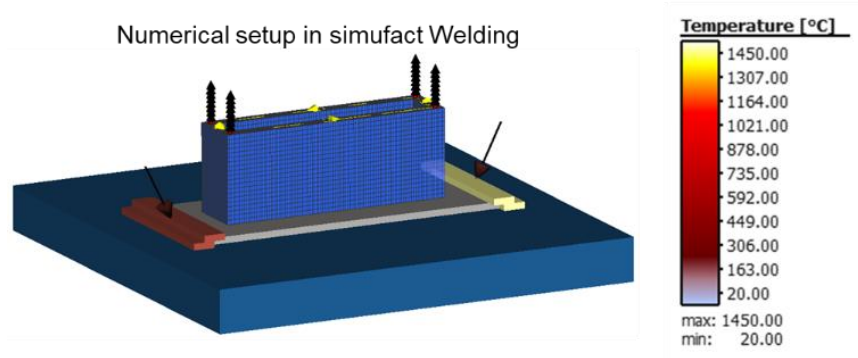

Hollow wall structure during welding process

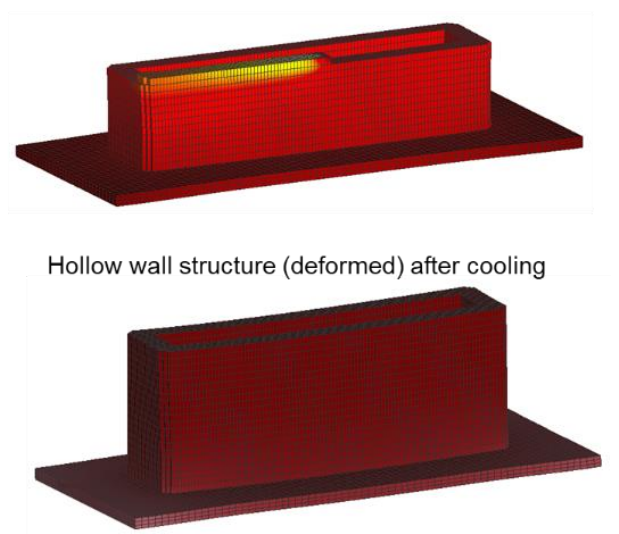

Figure 7. WAAM FEM simulation of a hollow wall structure

\subsection{CONCLUSION AND OUTLOOK}

Both Simufact Welding and Elmer FEM are suitable for performing professional FEM calculations. Nevertheless, there are significant differences that should be considered before selecting an FEM program. In direct comparison with Simufact, Elmer FEM is a very customizable FEM solver, which is particularly suitable for the academic application case. The Fortran programming interface can be used to implement not only different body forces, such as a moving heat source, but also custom solvers for new physical models. However, there is no ready-made welding library available. A so-called Solver Input File (SIF) provides Elmer's solvers with the necessary information. This SIF file is a simple text document that can be generated automatically if necessary to perform parameter studies. A weak point of Elmer FEM is the graphical user interface. In principle, Elmer simulations can be started from the terminal after creating a SIF file with a simple editor. The development of the graphical user interface is not the main focus of the developers. In addition, Elmer's pre- and post-processing capabilities are limited. For pre-processing, a meshing tool such as Gmsh should be used. For post-processing, ParaView [13] is the ideal complement to Elmer FEM. On the other hand, Simufact Welding provides a userfriendly interface, which can quickly be handled by experienced FEM-users. The graphical user interface is optimized for welding simulations and the program provides all of the features which are necessary for a complete welding setup, including a material database with specific welding related materials and properties. This makes Simufact Welding a very good tool for the specific conduction of welding FEM simulations especially in an industrial environment, where different welding technologies and various setups have to be established in short time intervals. An industry-oriented FEM welding tool requires licences, which obviously costs money and therefore also points out one significant advantage of Elmer over Simufact Welding. Elmer FEM is open source, extensible and can be used without any licensing costs, which makes it attractive especially for research institutions where welding simulations are not the key point of research. Additionally, the Professorship of Welding Engineering at the Chemnitz University of Technology is currently developing a welding library for Elmer FEM to make the simulation of arc welding processes easier. Elmer FEM is constantly being further developed. The capabilities and limitations of this software depend on the physical problem to be modelled. A detailed breakdown of the implemented solvers and software components including comprehensive documentation can be found on the official homepage of Elmer FEM [14].

\section{ACKNOWLEDGEMENT}

This research is supported by DAAD Germany with Project Code: 57525437 (Future Technology Additive Manufacturing).

\section{REFERENCES}

[1] T. A. Rodrigues, V. Duarte, R. M. Miranda, T. G. Santos, and J. P. Oliveira, "Current Status and Perspectives on Wire and Arc Additive Manufacturing (WAAM)," Materials (Basel)., vol. 12, no. 7, 2019, doi: 10.3390/ma12071121.

[2] F. Montevecchi, G. Venturini, A. Scippa, and G. Campatelli, "Finite Element Modelling of Wire-arcadditive-manufacturing Process," Procedia CIRP, vol. 55, pp. 109-114, 2016, doi: https://doi.org/10.1016/j.procir.2016.08.024.

[3] A. Razavykia, E. Brusa, C. Delprete, and R. Yavari, "An Overview of Additive Manufacturing Technologies-A Review to Technical Synthesis in Numerical Study of Selective Laser Melting," Materials (Basel)., vol. 13, no. 17, 2020, doi: 10.3390/ma13173895. 
[4] M. Graf, A. Hälsig, K. Höfer, B. Awiszus, and P. Mayr, "Thermo-Mechanical Modelling of Wire-Arc Additive Manufacturing (WAAM) of Semi-Finished Products," Metals (Basel)., vol. 8, no. 12, 2018, doi: 10.3390/met8121009.

[5] Y. Ling, J. Ni, M. Abdel Wahab, J. Antonissen, and J. Vande Voorde, “A Heat Transfer Finite Element Model for Wire-Arc-Additive-Manufacturing Process," in Proceedings of the 8th International Conference on Fracture, Fatigue and Wear, 2021, pp. 201-215.

[6] J. Ding, P. Colegrove, J. Mehnen, S. Williams, F. Wang, and P. S. Almeida, "A computationally efficient finite element model of wire and arc additive manufacture," Int. J. Adv. Manuf. Technol., vol. 70, no. 1, pp. 227-236, 2014, doi: 10.1007/s00170-013-5261-x.

[7] Prajadhiama, Keval P et al., "Development of Bead Modelling for Distortion Analysis Induced by Wire Arc Additive Manufacturing using FEM and Experiment," MATEC Web Conf., vol. 269, p. 5003, 2019, doi: 10.1051/matecconf/201926905003.

[8] H. Huang et al., "Stress and Distortion Simulation of Additive Manufacturing Process by High Performance Computing," 2018, vol. Volume 6A: Materials and Fabrication, doi: 10.1115/PVP201885045 .

[9] J. Jinlong, Z. Yue, D. Mingye, W. Aiping, and L. Quan, "Numerical simulation on residual stress and deformation for WAAM parts of alluminium alloy based on temperature funtion method," China Weld., vol. 29, no. 2, pp. 1-8, 2020, doi: 10.12073/j.cw.20191101002.

[10] "Gmsh: a three-dimensional finite element mesh generator with built-in pre- and post-processing." [Online]. Available: Gmsh, https://gmsh.info [Accessed May 28, 2021].

[11] "JMatPro: Material Simulation \& Calculation of Material Data." [Online]. Available: JMatPro, https://matplus.de/en/jmatpro/ [Accessed May 28, 2021].

[12] Simufact, "Infosheet Wärmequelle," 2016. [Online]. Available: Simufact, https://www.simufact.de/files/Medien/_1Anwendungsfelder/Welding/Simufact-InfosheetHeatSourceGeometry-SW5_de.pdf [Accessed May 28, 2021].

[13] "ParaView: Multi-platform data analysis and visualization application." [Online]. Available: Paraview, https://www.paraview.org/ [Accessed May 28, 2021].

[14] "Homepage of Elmer FEM." [Online]. Available: Elmer, https://www.csc.fi/web/elmer [Accessed Aug. 06, 2021]. 